\title{
Satisfação de crianças com a vida: as contribuições da família e da escola
}

\section{Children's Life Satisfaction: family's and school's contribution}

\section{Satisfacción de niños con la vida: las contribuciones de la familia y de la escuela}

\author{
Fabiane Friedrich Schütz* \\ Universidade Federal do Rio Grande do Sul - UFRGS, Porto Alegre, Rio Grande do \\ Sul, Brasil
}

\section{Marcela Bortolini**}

Universidade Federal do Rio Grande do Sul - UFRGS, Porto Alegre, Rio Grande do Sul, Brasil

\section{J orge Castellá Sarriera***}

Universidade Federal do Rio Grande do Sul - UFRGS, Porto Alegre, Rio Grande do Sul, Brasil

\begin{abstract}
RESUMO
O objetivo do estudo foi investigar a contribuição da satisfação com a família e com a escola para a predição da satisfação com a vida. Participaram 2105 crianças, com idades entre 10 e 12 anos matriculadas em escolas públicas e particulares do Rio Grande do Sul. Os instrumentos utilizados foram: âmbito satisfação com a escola do General Domain Satisfaction Index, cinco itens relativos à satisfação com aspectos dos relacionamentos familiares e a escala de item único Overal Life Satisfaction. Foram realizadas análises de variância por idade e sexo e análise de regressão múltipla. Os resultados indicam que existem diferenças estatisticamente significativas por idade e sexo. A análise de regressão múltipla verificou que os itens que mais predisseram a satisfação com a vida foram relativos a serem ouvidas pelos seus pais $(\beta=0,158 ; p<0,01)$, estarem satisfeitas com a experiência na escola $(\beta=0,156 ; p<0,01)$ e com os colegas $(\beta=0,124 ; p<0,01)$. Ressalta-se que a satisfação com a família e com a escola explicam $21,8 \%$ da variância da satisfação com a vida. Destaca-se a importância do estímulo ao diálogo entre família e escola para promover o bem-estar da criança.
\end{abstract}

Palavras-Chave: satisfação com a vida, escola, família, criança.

\section{ABSTRACT}

This study aimed to investigate the contribution of satisfaction with family and school to predict life satisfaction. Participated 2105 children from 10 to 12 years old, students of public and private schools located in a state at the south of Brazil, Rio Grande do Sul. The instruments were the satisfaction with school domain from the General Domain Satisfaction Index, five items regarding family satisfaction aspects and the single item Overal Life 
Satisfaction. Analyses of Variance by sex and age as well as Multiple Regression Analyses were performed. Results indicated significant differences by age and sex. Multiple Analyses Regression indicated that the items that most predicted life satisfaction were the items regarding to be listened by parents $(\beta=0,158 ; \quad p<0,01)$, to be satisfied with school experience $(\beta=0,156 ; p<0,01)$ and classmates $(\beta=0,124 ; p<0,01)$. It is noteworthy that satisfaction with family and school predicted $21,8 \%$ of variance of life satisfaction. It is important to point out that the dialogue between school and family should be continuous in order to promote children's well-being.

Keywords: life satisfaction, school, family, children.

\section{RESUMEN}

El objetivo del estudio fue investigar la contribución de la satisfacción con la familia y la escuela para la predicción de la satisfacción con la vida. Participó en 2105 los niños con edades entre 10 y 12 años matriculados en escuelas públicas y privadas de Río Grande do Sul. Los instrumentos utilizados fueron lo ámbito satisfacción con la escuela de lo General Domain Satisfaction Index, cinco ítems con respecto a la satisfacción con aspectos de las relaciones familiares y la escala de ítem unico Overal Life Satisfaction. Realizaran-se análisis de varianza para la edad y el sexo y el análisis de regresión múltiple. Los resultados muestran diferencias estadísticamente significativas para la edad y el sexo. El análisis de regresión múltiple mostró que los temas que más predicen la satisfacción con la vida se relacionan con ser escuchado por sus padres $(\beta=0,158 ; p<0,01)$, estar satisfechos con la experiencia en la escuela $(\beta=0,156 ; \quad p<0,01)$ y con sus compañeros $(\beta=0,124 ; p<0,01)$. Cabe señalar que la satisfacción con la familia y con la escuela predijeran $21,8 \%$ de varianza de satisfacción con la vida. Destaca-se la importancia de estimular el diálogo entre las familias y las escuelas para promover el bienestar de los niños.

Palabras clave: satisfacción con la vida, escuela, familia, niños.

\section{Introdução}

A satisfação com a vida é um componente cognitivo do bem-estar subjetivo. Refere-se a uma avaliação subjetiva e global sobre a vida que pode ser influenciada pelo ambiente, pelas experiências nos contextos e pelas etapas do desenvolvimento (Diener, 1994; 2009; 2012). Por levar em conta aspectos subjetivos em uma avaliação global, a satisfação com a vida é influenciada pela satisfação com diversos âmbitos (Diener, 1994). Investigar a avaliação que as pessoas fazem sobre os contextos, nos quais estão inseridas, pode contribuir para a compreensão do bem-estar (Casas, 2009). Neste estudo, compreende-se a satisfação com a vida a partir da ideia de que diferentes domínios contribuem para uma avaliação global sobre a vida (Huebner, 2004). Segundo o paradigma ecológico-contextual, para compreender o comportamento das pessoas é importante avaliar sua inserção nos sistemas e contextos pelos quais circula. As pessoas são melhor compreendidas quando consideradas parte de um 
contexto social multinivelado, multiestrutural e multideterminado (Kelly, 2006).

As avaliações que uma criança faz sobre sua vida diferem das avaliações de um adulto sobre a vida como um todo. O termo vida representa todas as áreas da vida de uma pessoa em um determinado tempo, ou um julgamento considerando todos os episódios desde o nascimento (Diener, 2005). Na infância, os principais contextos que podem influenciar a satisfação com a vida são a família e a escola (Moreno, Estévez, Murgui, \& Musitu, 2009). A satisfação nesses dois contextos é avaliada através da satisfação com pequenos aspectos de cada um desses âmbitos (Diener, 1994), tais como a satisfação com o lugar onde vive, com a escuta dos pais, com as notas na escola, com a experiência na escola, com os colegas, entre outros aspectos que contemplam a vida nesses ambientes.

Cardoso e Feres-Carneiro (2008) salientam que a família é vista como uma referência fundamental na vida, tanto no sentido de felicidade, suporte, promoção de equilíbrio pessoal, além de constituir um fator essencial na formação da subjetividade. Por ser uma das primeiras instâncias socializadoras na infância (Estevez, Murgui, Musito, \& Moreno, 2008), é o primeiro espaço a promover a aquisição de habilidades, comportamentos e valores (Oliveira, Siqueira, Dell'Aglio, \& Lopes, 2008), bem como o desenvolvimento da socialização, diante de um clima social positivo (Estevez et al., 2008). Além disso, as relações familiares contribuem para o desenvolvimento de relações de apego (Bowlby, 1997), e estão estritamente relacionadas ao desenvolvimento emocional saudável das crianças ao longo do seu desenvolvimento (Grusec \& Lytton, 1988; Parker, Tupling, \& Brown, 1997). Dessa forma, os pais configuram-se como figuras essenciais para promover na criança o senso de ser amada, validada, fonte de atenção e preocupação, aspectos estes associados a melhores vivências da criança no contexto da escola, das amizades, da família, bem como consigo mesma (Caminha \& Caminha, 2011).

A família desempenha um papel decisivo na educação formal e informal, pois a educação bem-sucedida da criança nesse núcleo servirá de apoio a sua criatividade e ao seu comportamento produtivo quando for adulto, especialmente porque é o ambiente no qual são absorvidos os valores morais e éticos (Gomes \& Pereira, 2005). Logo, a família ocupa um importante papel na vida das crianças e na maneira como passam a ver a escola e seu processo de escolarização (Santos, Nascimento, \& Menezes, 2012).

Quanto à satisfação com a escola, esta diz respeito a uma avaliação que envolve todas as experiências escolares (Huebner, Ash, \& Laughlin, 2001). Em estudo com o objetivo de verificar o quanto aspectos pessoais e fatores ecológicos e contextuais podem influenciar no bem-estar subjetivo de adolescentes, Oberle, SchonertReichl, \& Zumbo (2011) encontraram que relacionamentos pessoais 
positivos com pares e adultos na comunidade, além um senso de pertencimento à escola, associam-se positivamente com a satisfação com a vida. Com relação aos fatores que contribuem para a satisfação com a escola, destacaram o ambiente escolar e a complexidade dos fatores ambientais e pessoais. A conexão com a escola funciona como uma força protetora na adolescência e está positivamente relacionada com autoestima, desempenho escolar, motivação e ajustamento na escola (Oberle, Schonert-Reichl, \& Zumbo, 2011). Dotterer e Lowe (2011) buscaram encontrar as conexões entre o contexto de sala de aula, engajamento escolar e desempenho acadêmico entre adolescentes. Os resultados indicaram que o contexto escolar e o engajamento com a instituição funcionaram como preditores de desempenho acadêmico contribuindo para a satisfação com a escola.

Além disso, a capacidade de experimentar competências, ser valorizado pelos professores, tomar decisões e relacionar-se também estão associados ao bem-estar na escola (Hernangómez, Vázquez, \& Hervás, 2009). No ambiente de escolarização compreende-se que o clima social pode influenciar o desenvolvimento de crianças e adolescentes. O clima social escolar é baseado no grau de comprometimento com os estudos, nas relações entre pares e interações com professores e aspectos pessoais (Estevez et al., 2008).

Compreende-se que há uma relação importante entre a satisfação com a família, a satisfação com a escola e a satisfação com a vida. A infância é um período decisivo para a aprendizagem e o aprimoramento das habilidades sociais (Z. Del Prette \& Del Prette, 2005). A construção de um repertório socialmente habilidoso ocorre inicialmente entre pais e filhos, depois entre irmãos, e posteriormente entre colegas de escola e amigos (Gomide, 2003).

A avaliação de aspectos positivos da vida das crianças, tais como a satisfação com a vida, é um tema para o qual ainda existem poucos resultados empíricos, especialmente acessando dados subjetivos, como a avaliação sobre determinados domínios. Muitos dos estudos com crianças ainda consideram os adultos (pais e professores) os principais informantes (Casas, 2009; Huebner, 2004). É possível acessar indicadores objetivos de dados da infância, como taxas de mortalidade e escolaridade. Evidentemente, para os dados objetivos, os adultos são naturalmente consultados. Entretanto, para se obter indicadores subjetivos da infância é necessário que sejam estudadas as percepções das crianças sobre suas vidas (Ben-Arieh, 2005).

Para conhecer o que as crianças pensam e de que forma fazem essa avaliação elas devem ser sujeitos de pesquisa e consultadas sobre suas opiniões sobre a satisfação com os contextos. Noronha e Rodrigues (2011) pontuam que em estudos sobre saúde emocional de crianças, a opinião de seus pais é mais valorizada do que da 
própria criança. Com o passar dos anos, a incidência de investigações que priorizem a percepção da criança sobre o seu estado de saúde tem aumentado. Os pesquisadores compreendem que crianças a partir dos oito anos já possuem habilidades cognitivas que as capacitam para diferenciar as suas ideias das de outras pessoas e de expressá-las verbalmente (Noronha \& Rodrigues, 2011) e, portanto, acredita-se que as crianças de 10 a 12 anos possuem capacidade de avaliar suas vidas em diferentes domínios.

A satisfação com a vida refere-se a uma avaliação global sobre a vida e está relacionada com alguns domínios-chave. Hubner (2004) propõe que cinco domínios específicos podem contribuir para um fator único de satisfação com a vida na infância, dentre os quais a escola, família, amigos, self e ambiente de vida. O autor compreende que as principais experiências sociais ocorrem na escola e na família. Dessa forma, espera-se que ambas possam influenciar as percepções sobre amigos, self e ambientes.

Estudos anteriores já reportaram a importância de investigar a avaliação da satisfação com a vida através da percepção das próprias crianças, especialmente sobre os contextos com maiores contribuições para seu desenvolvimento, como a escola e a família (Huebner et al., 2001). Diante disso o objetivo do presente estudo foi investigar a contribuição da satisfação com a família e com a escola para a predição da satisfação com a vida.

\section{Método}

\subsection{Participantes}

Os participantes integram a amostra de um estudo prévio realizado pelo Grupo de Pesquisa em Psicologia Comunitária (GPPC), em parceria com a International Society for Child Indicators (ISCI). Essa investigação maior visa conhecer e comparar dados representativos da vida das crianças de diversos países.

A amostra do presente estudo é composta por 2105 crianças, com idades entre 10 e 12 anos $(M=10,87 ; D P=0,799)$, sendo $44 \%$ meninos e $56 \%$ meninas, regularmente matriculadas em escolas públicas $(59,9 \%)$ e privadas $(40,1 \%)$ do Rio Grande do Sul. As escolas participantes foram selecionadas por conveniência. As escolas que aceitaram a realização da pesquisa, formalizada pelo aceite através do Termo de Concordância Institucional $(\mathrm{TCl})$, tiveram seus alunos, do 3 o ao 60 ano e da $5^{\text {a }}$ a 7 ạ série, convidados a participar da pesquisa. Os alunos de todas as turmas dessas séries foram convidados pelos pesquisadores a responder ao questionário com as questões da pesquisa do GPPC e levaram para casa os Termos de Consentimento Livre e Esclarecido (TCLE). 
Apesar de todas as crianças terem sido convidadas a participar da pesquisa somente responderam aos questionários aquelas que trouxeram os TCLEs assinados pelos pais e por eles mesmos e os entregaram aos pesquisadores. A aplicação ocorreu coletivamente durante o período de aula, nas próprias salas, ou em salas indicadas pelos funcionários das escolas e teve duração aproximada de 40 minutos. A aplicação foi realizada por dois pesquisadores treinados.

\subsection{I nstrumentos}

Foi utilizado o questionário desenvolvido pela ISCI e pelo GPPC. O questionário tem por objetivo geral verificar a satisfação das crianças com contextos vitais (família, escola, comunidade, religião, ambiente), atividades diárias das crianças e uso do tempo. O questionário foi desenvolvido considerando a importância de apresentar uma linguagem adequada às crianças, em termos de apresentação das escalas. Foi realizada a tradução do instrumento do inglês e do espanhol, e, em seguida, foi submetido à back translation. Ocorreram duas aplicações piloto com o objetivo de verificar a compreensão das crianças com a formulação das questões e a compreensão semântica dos itens. Algumas palavras, de tradução literal, foram substituídas por sinônimos para melhor compreensão.

O questionário é composto por algumas escalas de bem-estar (OLS, PWI-SC, SLSS, BMSLSS), pelo índice General Domain Satisfaction Index (GDSI), e por itens específicos que avaliam a satisfação com diferentes domínios (vida familiar, recursos financeiros, bairro, escola, utilização do tempo).

Satisfação com a escola: Para avaliar a satisfação com esse domínio, foi utilizado um dos âmbitos do GDSI (Casas \& Bello, 2012). Esse âmbito é composto por 4 itens, que respondem à pergunta "O quanto você está satisfeito com": "a escola que você vai", "outras crianças da sua sala de aula", "suas notas na escola" e "a sua experiência na escola". A versão original do GDSI avalia a satisfação com a vida em oito âmbitos e possui um alpha de Cronbach de 0,90. Em estudo de comparação de crianças residindo com suas famílias e em situação de acolhimento, a escala apresentou alpha de Cronbach de 0,87 (Schütz, Sarriera, Bedin, \& Montserrat, 2014). A escala é do tipo Likert, de 11 pontos que varia de 0 (completamente insatisfeito) a 10 (completamente satisfeito). Para essa amostra o âmbito satisfação com a escola possui um índice de consistência interna aceitável, com alpha de Cronbach de 0,65.

Satisfação com a família: Esse domínio foi avaliado através de cinco itens relativos à satisfação com aspectos dos relacionamentos familiares presentes no questionário da ISCI e elaborado por essa equipe internacional. Esses itens foram incluídos no estudo devido a sua abrangência, uma vez que além de avaliar os relacionamentos 
familiares captam aspectos do ambiente familiar. Os itens são: “Eu me sinto seguro em casa", "Eu tenho um lugar tranquilo para estudar em casa", "Meus pais (ou as pessoas que escutam de mim) me escutam e levam em conta o que eu falo", "Nós temos bons momentos juntos em minha família", "Os meus pais (ou as pessoas que cuidam de mim) me tratam bem". Os itens foram medidos através de uma escala Likert de 5 pontos que varia de 0 (Discordo muito) a 4 (Concordo muito). Quando analisados em conjunto, os itens possuem boa consistência interna, com alpha de Cronbach de 0,72 .

Satisfação com a vida: a Overal Life Satisfaction (OLS) é item único que averigua a satisfação global com a vida, que varia de 0 (completamente insatisfeito) a 10 (completamente satisfeito). Campbel, Converse e Rodgers (1976) apontaram a importância da utilização de um item único na avaliação do bem-estar subjetivo. Originalmente, deve-se responder à, pergunta "Atualmente, até que ponto você está satisfeito com toda a sua vida, considerada globalmente?". Nesse estudo, essa questão teve a linguagem adaptada para as crianças e a pergunta final foi "O quanto satisfeito você se sente com a sua vida como um todo?".

\subsection{Procedimentos éticos}

Essa pesquisa foi previamente submetida ao Comitê de Ética em Pesquisa da Universidade. Após a aprovação foram solicitadas os TCls para a realização da pesquisa. Após as concordâncias das instituições foram entregues os TCLEs e somente participaram da pesquisa aquelas crianças que os trouxeram devidamente assinados pelos pais ou responsáveis.

\subsection{Procedimento de análise}

Inicialmente, foram apresentados os dados descritivos e realizadas duas Análises de Variância (ANOVAs) considerando sexo e idade. Em seguida, foi realizada Análise de Regressão Linear Múltipla para verificar a contribuição da satisfação com a família e com a escola para a predição da satisfação com a vida. Foi utilizado o método stepwise, o qual introduz uma variável por etapa, iniciando com aquela que explica a maior porcentagem da variável dependente. As variáveis independentes foram os itens relativos aos relacionamentos familiares e os itens relativos à escola. A variável dependente foi a OLS.

Para a utilização da Análise de Regressão Linear Múltipla foram verificados alguns pressupostos estatísticos recomendados por Hair et al. (2009), tais como linearidade, homoscedasticidade, normalidade, independência dos resíduos e multicolinearidade. A amostra 
apresentou linearidade e homoscedasticidade, verificadas através da análise do diagrama de dispersão. A normalidade foi verificada através da análise do histograma. A independência dos resíduos foi verificada através da estatística de Durbin-Watson cujo valor foi de 1,89, respeitando o grau de dependência dos resíduos. A multicolinearidade foi verificada através dos indicativos propostos por Field (2009). As correlações de Pearson foram moderadas (inferiores a 0,5$)$, os Fatores de Inflação de Variância ficaram em torno de 1 e os valores de tolerância ficaram acima de 0,7 .

\section{Resultados}

A seguir serão apresentadas, na Tabela 1, as médias das respostas das crianças para os itens dos instrumentos utilizados, considerandose a amostra por idade e sexo.

Tabela 1.

Médias e desvios-padrão dos instrumentos por idade e sexo

\begin{tabular}{|c|c|c|c|c|c|}
\hline \multirow{3}{*}{ Variáveis } & \multicolumn{3}{|c|}{ Idade } & \multicolumn{2}{|c|}{ Sexo } \\
\hline & 10 anos & 11 anos & 12 anos & Masculino & Feminino \\
\hline & \multicolumn{3}{|c|}{$M(D P)$} & \multicolumn{2}{|c|}{$M(D P)$} \\
\hline OLS & $\begin{array}{c}9,45 \\
(1,36)\end{array}$ & $\begin{array}{c}9,27 \\
(1,46)\end{array}$ & $\begin{array}{c}8,94 \\
(1,65)\end{array}$ & $\begin{array}{c}9,27 \\
(1,45)\end{array}$ & $\begin{array}{c}9,25 \\
(1,52)\end{array}$ \\
\hline $\begin{array}{l}\text { Satisfação com a } \\
\text { escola que você vai }\end{array}$ & $\begin{array}{c}9,29 \\
(1,63)\end{array}$ & $\begin{array}{c}9,11 \\
(1,76)\end{array}$ & $\begin{array}{c}8,79 \\
(1,85)\end{array}$ & $\begin{array}{c}8,98 \\
(1,86)\end{array}$ & $\begin{array}{c}9,19 \\
(1,64)\end{array}$ \\
\hline $\begin{array}{l}\text { Satisfação com } \\
\text { outras crianças da } \\
\text { sua sala de aula }\end{array}$ & $\begin{array}{c}8,41 \\
(2,07)\end{array}$ & $\begin{array}{c}8,27 \\
(2,18)\end{array}$ & $\begin{array}{c}8,01 \\
(2,10)\end{array}$ & $\begin{array}{c}8,22 \\
(2,20)\end{array}$ & $\begin{array}{c}8,28 \\
(2,06)\end{array}$ \\
\hline $\begin{array}{l}\text { Satisfação com suas } \\
\text { notas na escola }\end{array}$ & $\begin{array}{c}8,95 \\
(1,72)\end{array}$ & $\begin{array}{c}8,51 \\
(1,99)\end{array}$ & $\begin{array}{c}7,82 \\
(2,41)\end{array}$ & $\begin{array}{c}8,40 \\
(2,06)\end{array}$ & $\begin{array}{c}8,59 \\
(2,05)\end{array}$ \\
\hline $\begin{array}{l}\text { Satisfação com } \\
\text { experiência na } \\
\text { escola }\end{array}$ & $\begin{array}{c}9,22 \\
(1,47)\end{array}$ & $\begin{array}{c}9,10 \\
(1,58)\end{array}$ & $\begin{array}{c}8,63 \\
(1,86)\end{array}$ & $\begin{array}{c}8,86 \\
(1,79)\end{array}$ & $\begin{array}{c}9,16 \\
(1,48)\end{array}$ \\
\hline $\begin{array}{l}\text { Eu me sinto seguro } \\
\text { em casa }\end{array}$ & $\begin{array}{l}3,48 \\
(0,73)\end{array}$ & $\begin{array}{c}3,43 \\
(0,78)\end{array}$ & $\begin{array}{l}3,32 \\
(0,77)\end{array}$ & $\begin{array}{c}3,43 \\
(0,79)\end{array}$ & $\begin{array}{c}3,42 \\
(0,73)\end{array}$ \\
\hline $\begin{array}{l}\text { Eu tenho um lugar } \\
\text { tranquilo para } \\
\text { estudar em casa } \\
\text { Meus pais me }\end{array}$ & $\begin{array}{l}3,03 \\
(0,97)\end{array}$ & $\begin{array}{c}3,00 \\
(1,02)\end{array}$ & $\begin{array}{c}2,97 \\
(0,96)\end{array}$ & $\begin{array}{c}2,98 \\
(1,01)\end{array}$ & $\begin{array}{c}3,02 \\
(0,97)\end{array}$ \\
\hline $\begin{array}{l}\text { escutam e levam } \\
\text { em conta o que eu } \\
\text { falo }\end{array}$ & $\begin{array}{c}3,07 \\
(0,96)\end{array}$ & $\begin{array}{c}2,96 \\
(1,07)\end{array}$ & $\begin{array}{c}2,91 \\
(1,08)\end{array}$ & $\begin{array}{c}2,98 \\
(1,04)\end{array}$ & $\begin{array}{c}3,00 \\
(1,03)\end{array}$ \\
\hline $\begin{array}{l}\text { Nós temos bons } \\
\text { momentos juntos } \\
\text { em familia }\end{array}$ & $\begin{array}{c}3,52 \\
(0,78)\end{array}$ & $\begin{array}{c}3,46 \\
(0,86)\end{array}$ & $\begin{array}{c}3,32 \\
(0,87)\end{array}$ & $\begin{array}{c}3,44 \\
(0,84)\end{array}$ & $\begin{array}{c}3,46 \\
(0,83)\end{array}$ \\
\hline $\begin{array}{l}\text { Meus pais me } \\
\text { tratam bem }\end{array}$ & $\begin{array}{c}3,72 \\
(0,60)\end{array}$ & $\begin{array}{c}3,71 \\
(0,64)\end{array}$ & $\begin{array}{c}3,67 \\
(0,64)\end{array}$ & $\begin{array}{c}3,70 \\
(0,63)\end{array}$ & $\begin{array}{c}3,70 \\
(0,62)\end{array}$ \\
\hline
\end{tabular}


As médias dos itens de satisfação com a escola e de satisfação com a família diminuíram com o aumento da idade. Com relação ao sexo, as médias dos meninos são maiores do que as médias das meninas nos itens "Eu me sinto seguro em casa" e na OLS. Em todos os outros itens, as médias das meninas são maiores do que as dos meninos.

Foi realizada a Análise de Variância para verificar as diferenças entre idade (Tabela 2) e sexo (Tabela 3) por cada um dos itens.

Tabela 2.

Análise de Variância por idade

\begin{tabular}{|c|c|c|c|c|c|}
\hline & $\begin{array}{l}\text { Soma dos } \\
\text { quadrados }\end{array}$ & Gl & $\begin{array}{l}\text { Média dos } \\
\text { Quadrados }\end{array}$ & $\mathrm{F}$ & Sig. \\
\hline OLS & 75,768 & 2 & 37,884 & 17,286 & $0,000^{* * *}$ \\
\hline $\begin{array}{l}\text { Satisfação com a escola } \\
\text { que você vai }\end{array}$ & 70,260 & 2 & 35,130 & 11,679 & $0,000 * *$ \\
\hline $\begin{array}{l}\text { Satisfação com outras } \\
\text { crianças da sua sala de } \\
\text { aula }\end{array}$ & 44,559 & 2 & 22,280 & 4,944 & $0,007^{* * *}$ \\
\hline $\begin{array}{l}\text { Satisfação com suas notas } \\
\text { na escola }\end{array}$ & 370,530 & 2 & 185,265 & 45,711 & $0,000 * *$ \\
\hline $\begin{array}{l}\text { Satisfação com } \\
\text { experiência na escola }\end{array}$ & 101,647 & 2 & 5,824 & 19,496 & $0,000 * *$ \\
\hline $\begin{array}{l}\text { Eu me sinto seguro em } \\
\text { casa }\end{array}$ & 6,912 & 2 & 3,456 & 6,001 & $0,003 * *$ \\
\hline $\begin{array}{l}\text { Eu tenho um lugar } \\
\text { tranquilo para estudar em } \\
\text { casa }\end{array}$ & 1,484 & 2 & 0,742 & 0,765 & 0,466 \\
\hline $\begin{array}{l}\text { Meus pais me escutam e } \\
\text { levam em conta o que eu } \\
\text { falo }\end{array}$ & 8,107 & 2 & 4,054 & 3,826 & $0,022^{*}$ \\
\hline $\begin{array}{l}\text { Nós temos bons } \\
\text { momentos juntos em } \\
\text { familia }\end{array}$ & 10,927 & 2 & 5,464 & 7,822 & $0,000 * *$ \\
\hline Meus pais me tratam bem & 0,697 & 2 & 0,349 & 0,899 & 0,407 \\
\hline
\end{tabular}

$\mathrm{Na}$ variável idade, encontraram-se diferenças estatisticamente significativas na média da OLS, de todos itens de satisfação com a escola e nos itens "Eu me sinto seguro em casa", "Meus pais me escutam e levam em conta o que eu falo" e "Nós temos bons momentos juntos em família". Para verificar entre quais grupos de idade as crianças apresentaram diferenças foi realizado o teste post hoc de Bonferroni. Com relação aos itens "Satisfação com suas notas na escola" e à escala de item único OLS, as crianças apresentaram diferenças entre os 10 e 11 anos, 10 e 12 anos, e 11 e 12 anos. Entre os 10 e 12 e 11 e 12 anos, as crianças apresentaram diferenças nos itens "Eu me sinto seguro em casa" e "Nós temos bons momentos juntos em minha família", "Satisfação com a escola que você vai", "Satisfação com sua experiência na escola", "Satisfação com outras crianças na sua sala de aula". Já no item "Meus pais me escutam e 
levam em conta o que eu falo", as crianças diferem entre os 10 e 12 anos. Ressalta-se que as crianças apresentam, em todos os itens, médias maiores aos 10 anos e menores aos 12 anos. Já na variável sexo (Tabela 3), somente foram encontradas diferenças estatisticamente significativas entre as médias dos itens "Satisfação com a escola que você vai" e "Satisfação com experiência na escola".

Tabela 3.

Análise de Variância por sexo

\begin{tabular}{|c|c|c|c|c|c|}
\hline & $\begin{array}{l}\text { Soma dos } \\
\text { quadrados }\end{array}$ & $\mathrm{Gl}$ & $\begin{array}{l}\text { Média dos } \\
\text { Quadrados }\end{array}$ & $\mathrm{F}$ & Sig. \\
\hline OLS & 1,031 & 1 & 1,031 & 0,47 & 0,493 \\
\hline $\begin{array}{l}\text { Satisfação com a escola } \\
\text { que você vai }\end{array}$ & 16,391 & 1 & 16,391 & 5,449 & $0,020^{*}$ \\
\hline $\begin{array}{l}\text { Satisfação com outras } \\
\text { crianças da sua sala de } \\
\text { aula }\end{array}$ & 0,16 & 1 & 0,16 & 0,035 & 0,851 \\
\hline $\begin{array}{l}\text { Satisfação com suas notas } \\
\text { na escola }\end{array}$ & 9,825 & 1 & 9,825 & 2,424 & 0,120 \\
\hline $\begin{array}{l}\text { Satisfação com } \\
\text { experiência na escola }\end{array}$ & 34,533 & 1 & 34,533 & 13,247 & $0,000^{* *}$ \\
\hline $\begin{array}{l}\text { Eu me sinto seguro em } \\
\text { casa }\end{array}$ & 0,117 & 1 & 0,117 & 0,203 & 0,652 \\
\hline $\begin{array}{l}\text { Eu tenho um lugar } \\
\text { tranquilo para estudar em } \\
\text { casa }\end{array}$ & 1,262 & 1 & 1,262 & 1,3 & 0,254 \\
\hline $\begin{array}{l}\text { Meus pais me escutam e } \\
\text { levam em conta o que eu } \\
\text { falo }\end{array}$ & 0,051 & 1 & 0,051 & 0,048 & 0,827 \\
\hline $\begin{array}{l}\text { Nós temos bons } \\
\text { momentos juntos em } \\
\text { familia }\end{array}$ & 0,001 & 1 & 0,001 & 0,001 & 0,976 \\
\hline Meus pais me tratam bem & 0,076 & 1 & 0,076 & 0,196 & 0,658 \\
\hline
\end{tabular}

Em seguida, foi realizada a Análise de Regressão Múltipla (método stepwise) (Tabela 4) com o objetivo de investigar se as satisfações com a família e com a escola podem predizer a satisfação com a vida. Assim, os itens de satisfação com a família "Meus pais me escutam e levam em conta o que eu falo" e "Nós temos bons momentos juntos em família" e os itens de satisfação com a escola mostraram-se preditores da satisfação com a vida. O coeficiente ajustado de variância explicada $\left(R^{2}\right)$ foi de 0,218 , o que demonstra que o modelo é capaz de explicar $21,8 \%$ da variância dos itens de satisfação com a escola. 
Tabela 4

Variáveis preditoras de Satisfação com a Escola - modelo final

\begin{tabular}{|c|c|c|c|c|c|}
\hline \multirow{2}{*}{ Variáveis Preditoras } & \multicolumn{2}{|c|}{$\begin{array}{l}\text { Coeficientes não } \\
\text { padronizados }\end{array}$} & \multicolumn{3}{|c|}{$\begin{array}{l}\text { Coeficientes } \\
\text { padronizados }\end{array}$} \\
\hline & B & $\begin{array}{c}\text { Erro } \\
\text { padrão }\end{array}$ & $B$ & $T$ & Sig \\
\hline $\begin{array}{l}\text { Meus pais me escutam e } \\
\text { levam em conta o que } \\
\text { eu falo }\end{array}$ & 0,229 & 0,032 & 0,158 & 7,072 & 0,000 \\
\hline $\begin{array}{l}\text { Satisfação com a } \\
\text { experiência na escola }\end{array}$ & 0,142 & 0,022 & 0,156 & 6,551 & 0,000 \\
\hline $\begin{array}{l}\text { Satisfação com outras } \\
\text { crianças da sua sala de } \\
\text { aula }\end{array}$ & 0,108 & 0,016 & 0,155 & 6,758 & 0,000 \\
\hline $\begin{array}{l}\text { Nós temos bons } \\
\text { momentos juntos em } \\
\text { minha família }\end{array}$ & 0,227 & 0,040 & 0,127 & 5,718 & 0,000 \\
\hline $\begin{array}{l}\text { Satisfação com a escola } \\
\text { que você vai }\end{array}$ & 0,071 & 0,019 & 0,083 & 3,649 & 0,000 \\
\hline $\begin{array}{l}\text { Satisfação com as suas } \\
\text { notas na escola }\end{array}$ & 0,047 & 0,016 & 0,065 & 2,91 & 0,000 \\
\hline
\end{tabular}

As variáveis que mais predisseram a satisfação com a vida foram respectivamente "Meus pais me escutam e leva em conta o que eu falo" $(\beta=0,158 ; p<0,01)$, "Satisfação com a experiência na escola" $(\beta=0,156 ; p<0,01)$, "Satisfação com outras crianças da sua sala de aula" $(\beta=0,124 ; p<0,01)$. As seguintes variáveis que entraram no modelo de predição foram "Nós temos bons momentos juntos em minha família" $(\beta=0,127 ; p<0,01)$, "Satisfação com a escola que você vai" $(\beta=0,083 ; p<0,01)$ e "Satisfação com as suas notas na escola" $(\beta=0,067 ; p<0,01)$. As variáveis "Eu me sinto seguro em casa", "Eu tenho um lugar tranquilo para estudar em casa" e "Meus pais (ou as pessoas que cuidam de mim) me tratam bem" foram excluídas do modelo de predição.

\section{Discussão}

Os resultados apontam que as médias dos itens de satisfação com a escola, com a família e com a vida diminuíram com o aumento da idade. Outros estudos, com amostra de crianças e adolescentes brasileiros já demonstraram diminuição da satisfação com a vida, conforme o aumento da idade (Bedin \& Sarriera, 2014; Giacomoni \& Hutz, 2008). Esse resultado também vai ao encontro do estudo de Noronha e Rodrigues (2011), o qual verificou que crianças com maiores idades tendem a evidenciar menores índices de satisfação e realização do que as crianças mais novas. A tendência de diminuição 
da satisfação com a idade também foi encontrada na adolescência com amostra australiana (Tomyn \& Cummins, 2011) indicando a diminuição da satisfação em diversos domínios. Para Casas et al. (2012) esses resultados podem ser reflexo de um aspecto desenvolvimental que ainda não havia sido detectado nos estudos de bem-estar, visto que os estudos com crianças e adolescentes ainda são escassos.

Com a diminuição das médias dos itens relativos à escola, com a idade, pode-se pensar que a satisfação com a escola pode ser inversamente proporcional ao efeito estressor do aumento natural de exigências e responsabilidades aos quais as crianças vão sendo exigidas a medida que crescem. O estudo de Huebner et al. (2001) indica relações negativas entre ambientes estressores (com altos níveis de exigência de desempenho) e satisfação escolar.

Com relação às diferenças por sexo, encontrou-se que as meninas diferenciaram-se significativamente dos meninos, com médias superiores, nos itens "Satisfação com a escola que você vai" e "Satisfação com sua experiência na escola". Resultados da pesquisa do GPPC em parceria com a ISCI relativos às atividades que as crianças desenvolvem no tempo livre revelam que as meninas apresentam maior frequência para os comportamentos de ler, encontrar os amigos para estudar e fazer lição de casa (Sarriera et al., 2014). Além disso, nos estudos de Casas e Bello (2012) as meninas também apresentam maior satisfação com a escola.

Através da Análise de Regressão Múltipla verificou-se que os itens de satisfação com a família "Meus pais me escutam e leva em conta o que eu falo" e "Nós temos bons momentos juntos em minha família" e todos os itens de satisfação com a escola podem predizer $21,8 \%$ da variância da satisfação com a vida. Em consonância com a literatura, discute-se que a satisfação com a vida é influenciada por alguns domínios principais (Huebner, 2004), uma vez que alguns dos itens relativos à família e à escola podem predizer uma relevante parcela da satisfação com vida.

A variável "Meus pais me escutam e leva em conta o que eu falo" é a variável com maior capacidade preditiva. Crianças que são escutadas e consideradas em suas opiniões pelos seus cuidadores, possivelmente sentem-se acolhidas, validadas, respeitadas e fonte de atenção pelos seus pais. Segundo teóricos da psicologia do desenvolvimento, pais que estabelecem relações com seus filhos permeadas pela escuta, atenção, validação, trocas, auxiliam seus filhos a desenvolver um senso de pertencimento, de vínculo seguro e de aceitação, auxiliando no desenvolvimento emocional saudável da criança (Bowlby, 1979/1997); Grusec \& Lytton, 1988; Parker, Tupling \& Brown, 1979/1997; Young, Klosko \& Weishaar, 2008). Além disso, relações familiares que prezam pela comunicação, por meio da escuta e validação, reforçam o vínculo entre os membros (Bowlby, 1997). 
Diante disso, pode-se pensar que ser escutado pelos pais e estes levarem em conta, pode estar relacionado ao desenvolvimento de aspectos emocionais saudáveis, os quais possivelmente se associam à sensação de bem-estar com a vida.

Outra variável relativa à satisfação com a família que contribui para o modelo de predição de satisfação com vida foi o item "Nós temos bons momentos juntos em minha família". Em estudo qualitativo sobre a importância da família para o bem-estar de adolescentes, Schütz, Calza, Rodrigues e Sarriera (2014) encontraram que os adolescentes consideram a presença dos pais um dos aspectos que contribui para o bem-estar e que esse aspecto pode ser verificado através do tempo que passam juntos. A presença dos pais permite 0 fortalecimento dos vínculos e o estabelecimento de um clima familiar agradável (Schütz et al., 2014). A percepção da presença dos pais está relacionada à percepção de apoio familiar a qual influencia na capacidade de adaptação em diversos contextos na vida adulta (Paradis et al., 2009). Além de passar um bom tempo com os pais, outras variáveis relativas à uma boa convivência podem funcionar como um fator protetivo em outros contextos, assim como a comunicação dos pais com os filhos (Estevez et al., 2007).

Com relação à escola, verifica-se que todos os itens relativos a este contexto predisseram a satisfação com a vida. O item de satisfação com a escola com maior capacidade preditiva foi "Satisfação com a experiência na escola", seguido do item relativo à satisfação com outras crianças na sala de aula. Huebner et al. (2011) discutem que experiências extra-escolares e relacionamentos interpessoais são essenciais à satisfação escolar, conforme já evidenciado na literatura. Porém, esses autores encontraram que a experiência com a escola demonstrou-se tão importante quanto os relacionamentos interpessoais. No presente estudo, o mesmo ocorreu.

A satisfação com a escola que frequenta também contribuiu para a predição da satisfação com a vida. A percepção de satisfação com a instituição em si, está relacionada ao clima social escolar, uma vez que o clima escolar está relacionado com 0 grau de comprometimento com os estudos e relações interpessoais. Dotterer e Lowe (2011) encontraram o engajamento com a instituição escolar como preditor de desempenho acadêmico. Os relacionamentos interpessoais ligados ao clima escolar contribuem para a satisfação com a escola. Estevez et al. (2008) encontraram que pais, professores e amigos são as figuras mais importantes na infância e início da adolescência e que, o mundo social está diretamente relacionado com a avaliação que fazem sobre si mesmos, sobre os outros e o entorno.

O desempenho acadêmico, verificado através do item de satisfação com as notas, que entrou no modelo de predição, também está relacionado ao clima escolar e ao engajamento com a escola 
(Dotterer \& Lowe, 2011). Outra pesquisa também verificou a ligação entre a conexão e engajamento escolar com o desempenho acadêmico (Oberle et al., 2011).

As variáveis "Meus pais (ou as pessoas que cuidam de mim) me tratam bem", "Eu me sinto seguro em casa" e "Eu tenho um lugar tranquilo para estudar em casa" foram excluídas do modelo de predição. Quanto ao item sobre ser bem tratado pelos pais acreditase que a compreensão das crianças pode englobar aspectos diferentes daqueles concebidos pelos adultos. $O$ item pode abranger diversos aspectos da relação entre pais e filhos. A literatura identifica que alguns comportamentos paternos como apoio emocional, cuidado físico, suporte educacional e monitoramento tendem a estar associados ao bem-estar (Pople, Raws, Mueller, \& Mahony, 2014) e podem ser considerados aspectos de sentir-se bem tratado. Dessa maneira, é importante que se compreenda a concepção das crianças a respeito do significado de ser tratado bem.

Com relação a sentir-se seguro em casa, ressalta-se que os índices de violência dos centros urbanos vêm crescendo com frequência. A percepção das crianças com relação à segurança em casa pode estar relacionada aos altos índices de criminalidade. Ainda que, tanto a questão da segurança, quanto a questão de ter um lugar tranquilo para estudar não sejam preditoras de satisfação com a vida na infância podem ser explicadas quando se considera que as preocupações dos adultos, e influências sociais que os mesmos sofrem, são diferentes das preocupações das crianças (Casas \& Bello, 2012). Por conseguinte, para adultos, a segurança e as questões materiais relativas ao entorno apresentam-se como preditores de bem-estar (Diener, 2012), o que pode não se repetir na infância.

Ademais, os resultados também indicam que a escola, assim como a família, contribui para a satisfação com a vida. Todos os itens de escola analisados nessa pesquisa foram preditores de satisfação com a vida, em conjunto com dois itens de satisfação com a família. Assim, a escola e a família exercem mútua influência na avaliação que as crianças fazem sobre suas vidas. Oberle et al. (2011) encontraram que as interações sociais positivas em todos os contextos de desenvolvimento desempenham um papel essencial na satisfação com a vida de adolescentes e que, portanto, é importante para a satisfação com a vida que se desenvolvam relacionamentos saudáveis e interações ricas em todos os domínios da vida, especialmente na família e na escola. Esses resultados vão ao encontro dos achados da presente pesquisa que encontrou que tanto os itens de satisfação com a família quanto os itens de satisfação com a escola são importantes para a satisfação com a vida. Além disso, os resultados encontrados nesse estudo estão relacionados à percepção das crianças quanto aos contextos de relação mais próximos, o que facilita a compreensão da sua satisfação com a vida. 


\section{Considerações finais}

Este artigo contribui com a ideia de que a satisfação com a vida é influenciada por diferentes domínios, como a família e a escola e discute a importância dos mesmos para as avaliações das crianças. Os itens de escola e família conseguiram predizer em torno de $22 \%$ da satisfação com a vida das crianças participantes dessa pesquisa. Pode-se considerar que esses itens são responsáveis por explicar uma alta variância na satisfação com a vida das crianças, e que os profissionais que trabalham com a infância devem ter presente a influência da família e da escola, com as suas particularidades, para o bem-estar das crianças.

Acessaram-se as avaliações das crianças sobre suas vidas através de suas próprias percepções, adquirindo-lhes espaço para a participação na identificação de dados subjetivos que possam servir de subsídio para a construção de políticas das quais são objeto. A satisfação com a vida de crianças é um componente essencial para o conceito de bem-estar na infância, tão discutido em termos de políticas públicas.

As relações interpessoais, tanto na escola quanto na família, são cruciais para o desenvolvimento. A escola e a família exercem influência sobre o comportamento das crianças influenciando sua satisfação com a vida. Cabe às escolas a preocupação e consciência do seu papel na formação e na sua função como instância de socialização dos seus alunos, atentando para a literatura referente à promoção de saúde e de prevenção no ambiente escolar. As famílias que dispõem um tempo de qualidade com suas crianças estarão promovendo seu bem-estar. O diálogo e a parceria frente à tarefa educativa entre a escola e a família podem servir como um esforço conjunto para a promoção de um desenvolvimento mais saudável.

Considera-se ainda que a satisfação com a família, a satisfação com a escola e a satisfação com a vida podem ser mediadas por muitos fatores pessoais, ambientais e contextuais que não foram investigados nesse estudo. Ampliar o conhecimento sobre os diversos elementos que estão relacionados com a satisfação com a vida pode auxiliar na promoção de estratégias de intervenção psicossocial na infância. Também salienta-se que são necessárias pesquisas que investiguem como as crianças modificam e estabilizam suas percepções na escola e na família, e quais fatores podem influenciar a satisfação da criança em cada um dos âmbitos e contextos sociais.

Uma das limitações desse estudo é a utilização exclusiva de dados de questionários aplicados em formato tradicional que pode não captar a totalidade das especificidades que a pesquisa com crianças requer. Sugere-se que outros estudos possam complementar esses resultados com a utilização de desenhos, fotografias, grupos focais, 
linhas do tempo. Outra limitação desse estudo é a utilização de um corte transversal de idade. Estudos futuros longitudinais poderão especificar as relações entre idade e satisfação com a vida. Os resultados da presente pesquisa corroboram outros achados da literatura com relação à diminuição da satisfação com a vida com o aumento da idade e da importância da satisfação com a família e com a escola para a satisfação com a vida.

\section{Referências}

Bedin, L. M., \& Sarriera, J. C. (2014). Dyadic Analysis of ParentChildren Subjective Well-Being. Child Indicators Research, 7(3), 613-631.

Ben-Arieh, A. (2005). Where are the children? Children's role in measuring and monitoring their well-being. Social Indicators Research, 74(3), 573-596.

Bowlby, J. (1997). Formação e rompimento dos laços afetivos (3a ed.). São Paulo: Martins Fontes. (Trabalho original publicado em 1979).

Caminha, R.C., \& Caminha, M. (2011). Intervenções e treinamento de pais na clínica infantil. Sinopys.

Cardoso, C. L., \& Féres-Carneiro, T. F. (2008). Sobre a família: com a palavra, a comunidade. Estudos e Pesquisas em Psicologia, $8(2), 511-526$.

Casas, F. (2009). El bienestar personal: Su investigación en la infancia y la adolescencia. Encuentros en Psicología Social, 5(1), 85-101.

Casas, F., \& Bello, A. (2012). Calidad de vida y bienestar infantil subjetivo en España Madrid: Unicef.

Casas, F., Coenders, G., González, M., Malo, S., Bertran, I., \& Figuer, C. (2012). Testing the relationship between parents' and their children's subjective well-being. Journal of Happiness Studies, 13(6), 1031-1051. doi: 10.1007/s10902-011-9305-3.

Del Prette, Z. A. P. \& Del Prette, A. (2005). Psicologia das habilidades sociais na infância: teoria e prática. Petrópolis: Vozes.

Diener, E. (1994). Assessing subjective wellbeing: progress and opportunities. Social Indicators Research 31(2), 103-157.

Diener, E. (2005). Guidelines for National Indicators of Subjective Well-Being and III-Being. Chicago: University of Illinois.

Diener, E. (2009). Subjective well-being. Social Indicators Research Series, 37(1), 11-58.

Diener, E. (2012). New findings and future directions for subjective well-being research. American Psychologist, 67(8), 590-597. 
Dotterer, A.M., \& Lowe, K. (2011). Classroom Context, School Engagement, and Academic Achievement in Early Adolescence. Journal of Youth and Adolescence, 40(12), 1649-1660.

Estévez, E., Murgui, S., Moreno, D., \& Musitu, G. (2007). Estilos de comunicación familiar, actitud hacia la autoridad institucional y conducta violenta del adolescente en la escuela. Psicothema, 19(1), 108-113.

Estevez, E.L., Murgui, S.P., Musitu, G.O., \& Moreno, D.R. (2008). Clima familiar, clima escolar y satisfacción con la vida en adolescentes. Revista Mexicana de Psicologia, 25(1), 119-128.

Field, A. (2009). Descobrindo a estatística usando o SPSS. Porto Alegre: Artmed.

Giacomoni, C. (2002). Bem-estar subjetivo infantil: conceito de felicidade e construção de instrumentos para avalição. Tese de Doutorado, Programa de Pós Graduação em Psicologia, Universidade Federal do Rio Grande do Sul, RS, Brasil.

Giacomoni, C. H., \& Hutz, C. S. (2008). Escala multidimensional de satisfação de vida para crianças: estudos de construção e validação. Estudos de Psicologia (Campinas), 25(1), 25-35.

Gomes, M. A., \& Pereira, M. L. D. (2005). Família em situação de vulnerabilidade social: uma questão de políticas públicas. Ciências \& Saúde Coletiva, 10(2), 357-363.

Gomide, P. I. C. (2003). Estilos parentais e comportamento antissocial. In: A. Del Prette \& Z. A. P. Del Prette (orgs.). Habilidades sociais, desenvolvimento e aprendizagem: questões conceituais, avaliação e intervenção. (pp. 21-60). Campinas: Alínea.

Grusec, J. E. \& Lytton, H. (1988). Social development: History, theory and research. New York: Springer-Verlang.

Hair, J.F.J., Black, W.C., Babin, B.J., Anderson, R.E., \& Tatham, R.L. (2009). Análise Multivariada de Dados. Porto Alegre: Artmed.

Huebner, E. D. (2004). Research on assessment of life satisfaction of children and adolescents. Social Indicators Research, 66(1).

Huebner, E. S. Asher, C., \& Laughlin, J. E. (2001). Life experiences, locus of control, and school satisfaction in adolescence. Social Indicators Research, 55(1), 167-183.

Hernangómez, L., Vázquez, C., \& Hérvaz, G. (2009). El paisage emocional a lo largo de La vida. In. C. Vazquez \& G. Hérvas (Eds). La ciencia del bienestar: fundamentos de uma psicologia positiva. Madrid: Alianza Editorial.

Kelly, J. G. (2006). Becoming ecological: an expedition into community psychology. New York: Oxford Press.

Moreno, D. R., Estévez, E .L., Murgui, S. P., \& Musitu, G. O. (2009). Relación entre el clima familiar y el clima escolar: el rol de la empatía, la actitud hacia la autoridad y la conducta violenta en 
la adolescencia. International Journal of Psychology and Psychological Therapy, 9(1), 123-136.

Noronha, M. S., \& Rodrigues, M. A. (2011). Saúde e bem-estar de crianças em idade escolar. Escola Anna Nery, 15(2), 395-401.

Oliveira, D., Siqueira, A., Dell'Aglio, D., \& Lopes, R. (2008). Impacto das configurações familiares no desenvolvimento de crianças e adolescentes: Uma Revisão da Produção Científica. Interação em Psicologia, 12(1), 87-98.

Oberle E., Schonert-Reichl, K. A., \& Zumbo, B. Z. (2011). Life Satisfaction in Early Adolescence: Personal, Neighborhood, School, Family, and Peer Influences. Journal of Youth and Adolescence, 40(7), 889-901.

Paradis, A., Giaconia, R.M., Reinherz, H.Z., Beardslee, W.R., Ward, K.E., \& Fitzmaurice, G.M. (2009). Adolescent Family Factors, Promoting Healthy Adulct Functioning: A Longitudinal Community Study. Child and Adolescent Mental Health, 16(1), 30-37.

Parker, G., Tupling, H., \& Brown, L. B. (1979). A Parental Bonding Instrument. British Journal of Medical Psychology, 52, 1-10.

Pople, L., Raws, P. Mueller, D. \& Mahony, S. (2014). The Good Childhood Report 2014. The Children's Society: London.

Sarriera, J.C., Schütz, F.F., Galli, F., Bedin, L., Strelhow, M.R., \& Calza, T. Z. (2014). Bem-Estar na infância e fatores psicossociais associados. Porto Alegre: Concórdia.

Schütz, F. F. Calza, T. Z. Rodrigues, A. L., \& Sarriera, J. C. (2014). A percepção de adolescentes sobre a influência dos relacionamentos familiares no Bem-Estar In Garcia, A., Pereira, F.N., \& Oliveira, M.S.P. Relações Interpessoais e Sociedade. Vitória: CIPRI/UFES.

Schütz, F. F., Sarriera, J. C., Bedin, L. M., \& Montserrat, C. (2014). Subjective well-being of children in residential care centers: Comparison between children in institutional care and children living with their families. Psicoperspectivas, 15(1), 19-30.

Santos, R. M., Nascimento, M. A., \& Menezes, J. A. (2012). Os sentidos da escola pública para jovens pobres da cidade de Recife. Revista Latino Americana de Ciencias Sociales, Niñez y Juventud, 13(1), 280-300.

Tomyn, A. J., \& Cummins, R. A. (2011). The subjective wellbeing of high-school students: Validating the Personal Wellbeing IndexSchool Children. Social Indicators Research, 101(3), 405-418. doi: 10. 1007/s11205-010-9668-6.

Young, J. E., Klosko, J. S., \& Weishaar, M. E. (2008). Terapia do esquema: Guia de técnicas cognitivo-comportamentais inovadoras. Porto Alegre: Artmed. 


\section{Endereço para correspondência}

Fabiane Friedrich Schütz

Universidade Federal do Rio Grande do Sul - UFRGS, Pós Graduação em Psicologia

Rua Ramiro Barcelos, 2600, Santa Cecília, CEP 90035-003, Porto Alegre - RS, Brasil

Endereço eletrônico: fabianefschutz@gmail.com

\section{Marcela Bortolini}

Universidade Federal do Rio Grande do Sul - UFRGS, Pós Graduação em Psicologia

Rua Ramiro Barcelos, 2600, Santa Cecília, CEP 90035-003, Porto Alegre - RS, Brasil

Endereço eletrônico: bortolini.marcela@gmail.com

\section{J orge Castellá Sarriera}

Universidade Federal do Rio Grande do Sul - UFRGS, Pós Graduação em Psicologia Rua Ramiro Barcelos, 2600, Santa Cecília, CEP 90035-003, Porto Alegre - RS, Brasil

Endereço eletrônico: jorgesarriera@gmail.com

Recebido em: 19/01/2015

Reformulado em: 16/03/2016

Aceito para publicação em: 05/05/2016

\section{Notas}

* Psicóloga. Doutoranda e Mestre em Psicologia pelo Programa de Pós Graduação em Psicologia da Universidade Federal do Rio Grande do Sul.

** Psicóloga. Doutoranda e Mestre em Psicologia pelo Programa de Pós Graduação em Psicologia da Universidade Federal do Rio Grande do Sul.

*** Psicólogo. Pós Doutor em Técnicas Estatísticas Multivariadas (Universitat de Barcelona) e em Psicologia Comunitária (University of San Francisco). Doutor em Psicologia pela Universidade Autônoma de Madrid. Professor associado no Instituto de Psicologia da Universidade Federal do Rio Grande do Sul e Coordenador do Grupo de Pesquisa em Psicologia Comunitária. 